\title{
Sazhinite-(La), $\mathrm{Na}_{3} \mathrm{LaSi}_{6} \mathrm{O}_{15}\left(\mathrm{H}_{2} \mathrm{O}\right)_{2}$, a new mineral from the Aris phonolite, Namibia: Description and crystal structure
}

\author{
F. Cámara ${ }^{1} *$, L. Ottolini ${ }^{1}$, B. Devouard ${ }^{2}$, L. A. J. Garvie ${ }^{3}$ and F. C. Hawthorne ${ }^{4}$ \\ ${ }^{1}$ CNR-Istituto di Geoscienze e Georisorse, Sede di Pavia, via Ferrata 1, I-27100 Pavia, Italy \\ ${ }^{2}$ Département des Sciences de la Terre, Université Blaise Pascal, UMR 6524 CNRS-UBP-OPGC "Laboratoire \\ Magmas et Volcans", 5 rue Kessler, F-63038 Clermont-Ferrand, France \\ ${ }^{3}$ Department of Geological Sciences, Arizona State University, Tempe AZ 85287-1404, USA \\ ${ }^{4}$ Department of Geological Sciences, University of Manitoba, Winnipeg, Manitoba R3T 2N2, Canada
}

\section{ABSTRACT}

Sazhinite-(La) is a new mineral from the Aris phonolite, Windhoek, Namibia. It occurs in vesicles within the phonolite, together with other species crystallized from late-stage hydrothermal fluids: natrolite, aegirine, microcline, apophyllite, sphalerite, analcime, fluorite, villiaumite, hydroxylapatite, galena, makatite, quartz, eudialyte, kanemite, tuperssuatsiaite and korobitsynite. Sazhinite-(La) forms small euhedral crystals up to $1 \mathrm{~mm}$ long and $0.4 \mathrm{~mm}$ wide, elongated along [001] and flattened on (010), exhibiting the forms $\{h 0 l\},\{100\}$ and $\{001\}$. It has good cleavage parallel to $\{010\}$ and $\{001\}$. Twinning was not observed. Crystals are brittle with a Mohs hardness of 3, creamy white with a white streak, vitreous to pearly lustre, and translucent to transparent. In plane-polarized light, crystals are colourless with $a=Z, b=Y, c=X$. It is biaxial positive with $\alpha=1.524, \beta=1.528, \gamma=1.544$, all $\pm 0.002,2 \mathrm{~V}_{Z}(\mathrm{obs})=46(1)^{\circ}$, and $2 \mathrm{~V}_{Z}$ (calc. $)=53.6^{\circ}$.

Sazhinite-(La) is orthorhombic Pmm2, $a=7.415(2), b=15.515(3), c=7.164(1) \AA$, and $V=$ $824.2 \AA^{3}$. One crystal was studied by X-ray diffraction, electron microprobe and secondary ion mass spectrometry (SIMS) microanalysis, leading to the average composition $\left(\mathrm{Na}_{2.87} \mathrm{~K}_{0.02} \mathrm{Sr}_{0.01}\right)_{\Sigma 2.90}$ $\left[\mathrm{La}_{0.41} \mathrm{Ce}_{0.35} \mathrm{Pr}_{0.02} \mathrm{Nd}_{0.04}(\mathrm{Sm}, \mathrm{Gd}, \mathrm{Dy}, \mathrm{Er}, \mathrm{Yb})_{\Sigma 0.01} \mathrm{Th}_{0.09} \mathrm{U}_{0.01} \mathrm{Y}_{0.01} \mathrm{Zr}_{0.01} \mathrm{Ca}_{0.08} \mathrm{Li}_{0.01}\right]_{\Sigma 1.04}{ }^{\mathrm{T}}\left(\mathrm{Si}_{5.87} \mathrm{~S}_{0.06} \mathrm{~B}_{0.01}\right)$ $\left(\mathrm{O}_{14.86} \mathrm{~F}_{0.14}\right) \cdot\left(\mathrm{H}_{2} \mathrm{O}\right)_{2}$.

Weighted full-matrix least-squares refinement on 3369 reflections yielded $R_{\text {all }}=3.8 \%$. The structure is built of corrugated $\left[\mathrm{Si}_{6} \mathrm{O}_{15}\right]^{6-}$ layers linked by [7]-coordinated $R E E$ and $R^{4+}$ cations. This framework leaves channels that contain three [5]- and [6]-coordinated $\mathrm{Na}$ cations per formula unit that compensate for the residual charge on the silicate layers. The SIMS analyses confirm a $\mathrm{Na}$ content of 3 atoms per formula unit, leading to an ideal formula of $\mathrm{Na}_{3} \mathrm{LaSi}_{6} \mathrm{O}_{15}\left(\mathrm{H}_{2} \mathrm{O}\right)_{2}$. The third $\mathrm{Na}$ atom is bonded to $\mathrm{H}_{2} \mathrm{O}$ groups and therefore the total content of both $\mathrm{Na}$ and $\mathrm{H}_{2} \mathrm{O}$ may be reduced to 2 and 1 per formula, respectively. The depletion in Na allows for the entrance of high-charge cations such as $\mathrm{Th}^{4+}$.

KeYwords: sazhinite-(La), new mineral, EMPA, SIMS, SCXRD, rare-earth minerals.

\section{Introduction}

Sazhinite- $(\mathrm{CE}), \mathrm{Na}_{3} \mathrm{CeSi}_{6} \mathrm{O}_{14}(\mathrm{OH}) \cdot 6\left(\mathrm{H}_{2} \mathrm{O}\right)$, was first described from the Yubileinaya alkaline pegmatite, Mt. Karnasurt, Lovozero massif, Kola Peninsula, Russia (Es'kova et al., 1974). Since then, it has been reported from the Poudrette quarry, Mont Saint-Hilaire, Québec, Canada

*E-mail: camara@crystal.unipv.it

DOI: $10.1180 / 0026461067040343$
(Horváth and Gault, 1990, Chao et al., 1991) and from the Demix-Varennes quarry, SaintAmable sill, Varennes, Verchéres Co., Québec, Canada (Horváth et al., 1998). Sazhinite-(Ce) is orthorhombic, Pmm2, $a=7.50(3), b=15.62(6), c$ $=7.35(3) \AA, V=861 \AA^{3}$, with the general formula $\mathrm{H}_{x} \mathrm{Na}_{3-x} \mathrm{CeSi}_{6} \mathrm{O}_{15} \cdot n\left(\mathrm{H}_{2} \mathrm{O}\right)(n \geqslant 1.5)$ (Shumyatskaya et al. 1980). These authors detected $\mathrm{H}_{2} \mathrm{O}$ groups at the $\mathrm{O}(12)$ and $\mathrm{O}(13)$ sites but questioned the feasibility of the $\mathrm{O}(13)$ position because of the high isotropic atom- 
displacement parameter found in the refinement $\left(10.02 \AA^{2}\right)$. Here, we report the occurrence of a new mineral species, sazhinite-(La) (IMACNMMN 2002-42a) and a new occurrence of sazhinite-(Ce). This work is dedicated to the memory of David Shannon, who passed away on 2nd January, 2004. Dave collected large amounts of material from Aris in 1996, and provided the samples which allowed the description of this new species.

\section{Occurrence}

Sazhinite occurs in the Aris phonolite, located $25 \mathrm{~km}$ south of Windhoek, Namibia. Samples were collected from a quarry being mined for road and railway ballast. The phonolite is highly vesicular, fine-grained, aphyric and consists of alkali feldspars, nepheline and acmite, with minute accessory apatite, zircon and monazite (von Knorring and Franke, 1987). The vesicles in our samples range from $<1 \mathrm{~mm}$ to $>10 \mathrm{~cm}$ in diameter. Many are filled with fluid and appear to 'burst' when the rocks are split. In addition to rare sazhinite, the vesicles commonly contain natrolite, aegirine, microcline, apophyllite, sphalerite, analcime, fluorite, villiaumite, hydroxylapatite, galena, makatite, quartz, eudialyte (von Knorring and Franke, 1987), kanemite (Garvie et al., 1999), tuperssuatsiaite (von Knorring et al., 1992; Cámara et al., 2002), and korobitsynite (Niedermayr et al., 2002).

\section{Physical and optical properties}

Sazhinite-(La) occurs as euhedral crystals up to $1 \mathrm{~mm}$ long and $0.4 \mathrm{~mm}$ wide, although neither end of any crystal was observed (Fig. 1). Crystals are elongated along [001] and flattened on (010). The most common forms are the dome $\{h 01\}$ and the pinacoids $\{100\}$ and $\{010\}$. Twinning was not observed. Cleavage planes $\{010\}$ and $\{001\}$ are good and crystals are brittle. The larger crystals are commonly altered. Unaltered sazhinite crystals are creamy white with a white streak, with a vitreous to pearly lustre. The density, based on the crystal analysed (see below), is $2.64 \mathrm{~g} / \mathrm{cm}^{3}$.

Sazhinite-(La) is colourless in transmitted light, optically biaxial positive, with $\alpha=1.524 \pm 0.002, \beta$ $=1.528 \pm 0.002$ and $\gamma=1.544 \pm 0.002(\lambda=$ $589 \mathrm{~nm}$ ), and birefringence of 0.020 . From spindle stage data (using the software by Bartelmehs et al., 1992), $2 \mathrm{~V}_{\mathrm{Z}}$ (observed) is $46(1)^{\circ} ; 2 \mathrm{~V}_{Z}$ (calculated) $=53.6^{\circ}$. Orientation:
$a=Z, b=Y, c=X$, and the optic axial plane coincides with (010). The compatibility index (Mandarino, 1981) is -0.025 (excellent).

\section{Chemical composition}

\section{Electron microprobe analysis (EMPA)}

Two sazhinite crystals were embedded in an epoxy mount and analysed using a Cameca SX100 electron microprobe. Natural and synthetic compounds were used as standards. Analytical conditions and procedures wee reported by Ottolini et al. (2004), where problems related to beam damage and $\mathrm{Na}$ mobility during EMPA are also discussed. We measured systematically low totals corresponding to difficulties in analysing $\mathrm{Na}$ (Ottolini et al., 2004).

The locations of the points analysed in crystals 1 and 2 are shown in Fig. 2 and the chemical compositions and overall analytical uncertainties are reported in Table 1 . In addition, qualitative X-ray maps (Fig. 3) were recorded using a Jeol JSM-5910LV SEM fitted with a PGT EDS microanalysis system operating at an accelerating voltage of $15 \mathrm{kV}$ and $1 \mathrm{nA}$ probe current. Acquisition time was $7600 \mathrm{~s}$ (dwell time $4.9 \times 10^{-4}$ s/pixel per frame, averaging 70 frames), collecting $14000 \mathrm{cps}$ in a total spectrum of 512 pixel-sized maps. The emission lines used were $\mathrm{O} K \alpha$, Si $K \alpha$, Na $K \alpha$, La $L \alpha_{1}$, Ce $L \alpha_{1}$, and Th $M \alpha$, counting in energy windows centred on the lines and 1.5 FWHM wide.

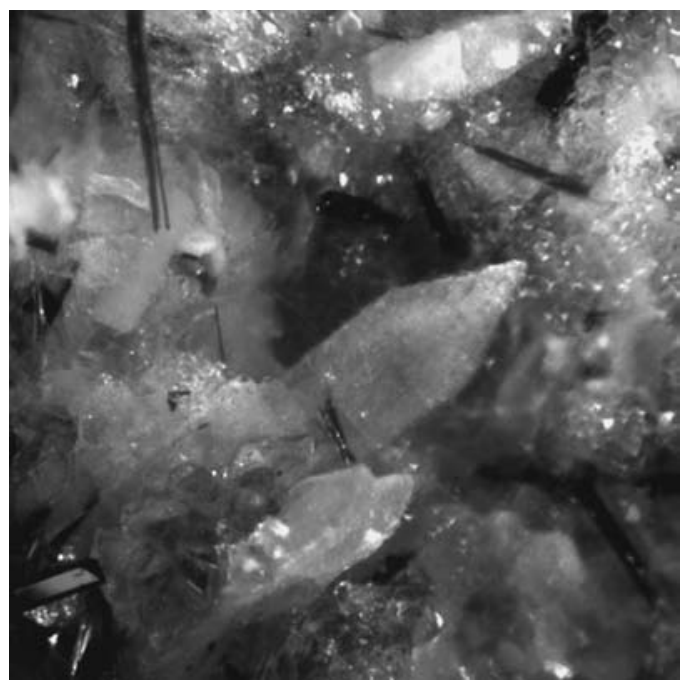

FIG. 1. Sazhinite crystal in a vug. The sazhinite crystal is $\sim 0.6 \mathrm{~mm}$ long. 

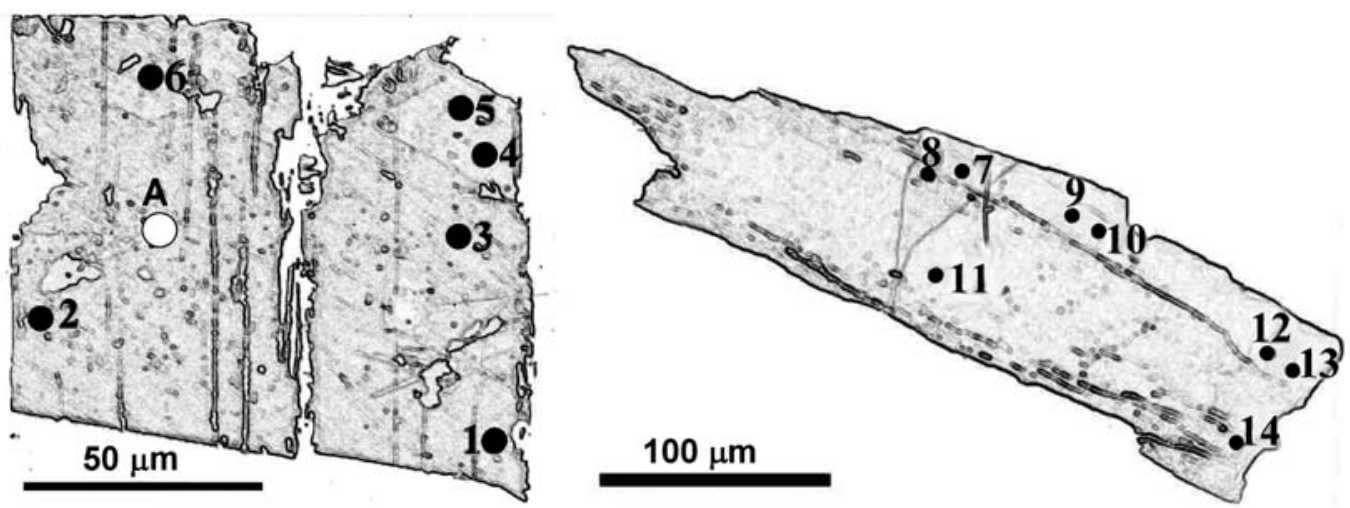

FIG. 2. Sketches of the two sazhinite grains used in this study showing the locations of the points analysed for Tables $1(1-14)$ and $2(\mathrm{~A})$.

Significant $\mathrm{S}$ contents were recorded by EMPA, and show a negative correlation with $\mathrm{Si}$. This, together with the problems related to low $\mathrm{Na}_{2} \mathrm{O}$, led us to re-normalize the EMPA data on the basis of $(\mathrm{Si}+\mathrm{S})=6$ atoms per formula unit (a.p.f.u., Table 1). Table 1 shows the analysed and stoichiometric values for $\mathrm{Na}_{2} \mathrm{O}$, together with stoichiometric value for $\mathrm{H}_{2} \mathrm{O}$ derived from the structure refinement. The different chemical compositions reported in Table 1 correlate with the back-scattered electron (BSE) images (Figs 2 and 3) and X-ray maps. In particular, $\mathrm{La}_{-1} \mathrm{Th}$ is the main chemical exchange in both crystals (Fig. 4a). Crystal 1 (used for the X-ray single crystal diffraction study) is dominantly sazhinite(La) with a Th-rich rim. Crystal 2 is sazhinite(Ce) with minor Th depletion towards the rim and a region of Th-depleted sazhinite-(La) (analysis points 7 and 8 , Fig. $3 b$ ). In order to maintain local charge-balance, such an exchange implies equal quantities of $\mathrm{Ca}$ and $\mathrm{Th}$. This is not the case as $\mathrm{Ca}$ and Th are inversely correlated (Fig. 4b),
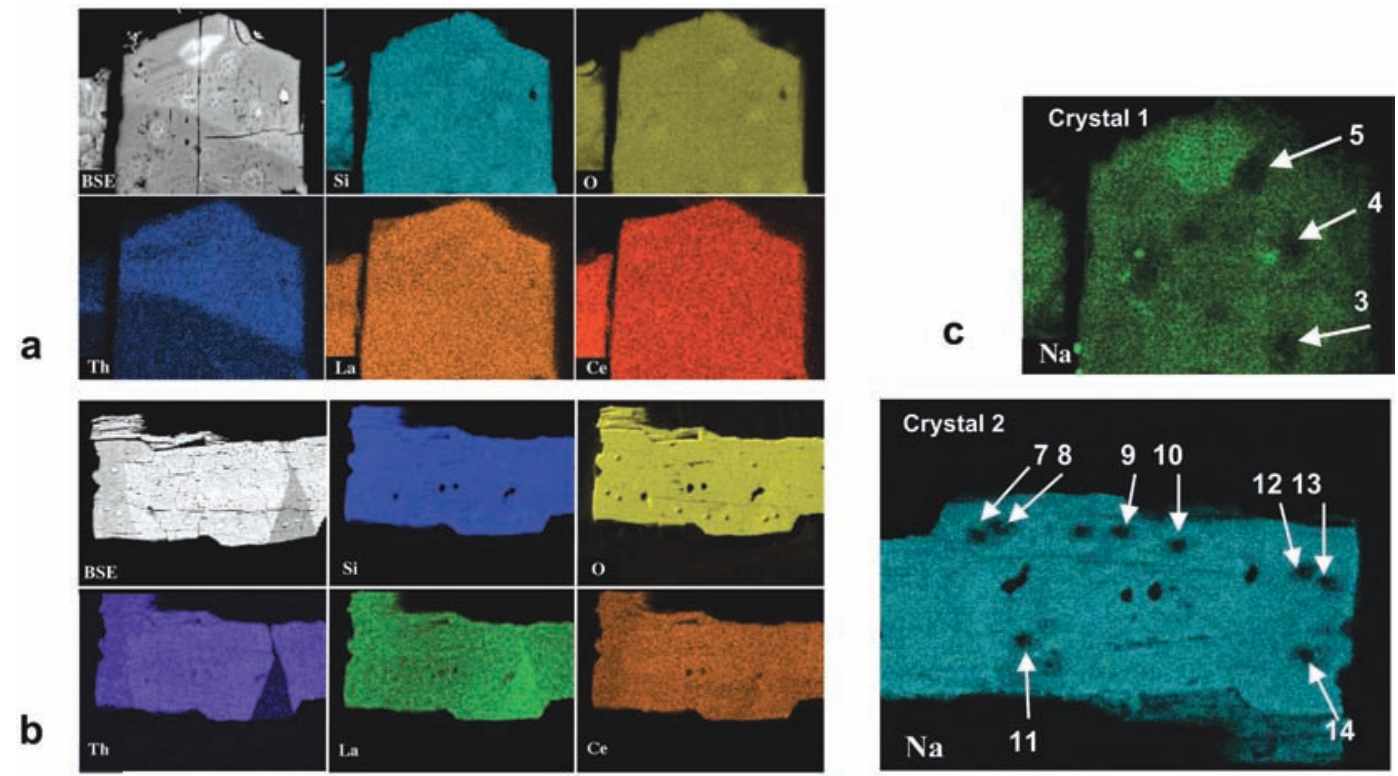

FIG. 3. Back-scattered electron (BSE) images and X-ray maps of (a) crystal 1 and (b) crystal 2. (c) Na X-ray maps of crystals 1 and 2 showing beam damage after EMP analysis. 
TABLE 1a. Electron microprobe analyses and formulae on the basis of $\mathrm{Si}+\mathrm{S}=6$ a.p.f.u. for crystal 1.

\begin{tabular}{|c|c|c|c|c|c|c|c|c|}
\hline & 1 & & 2 & & 3 & 4 & 5 & 6 \\
\hline $\mathrm{SiO}_{2}$ & $54.47 \pm 1.44$ & 53.95 & \pm 1.43 & 53.54 & \pm 1.42 & $51.26 \pm 1.37$ & $51.93 \pm 1.38$ & $52.95 \pm 1.41$ \\
\hline $\mathrm{La}_{2} \mathrm{O}_{3}$ & $11.55 \pm 0.25$ & 11.09 & \pm 0.24 & 10.31 & \pm 0.23 & $9.95 \pm 0.22$ & $10.27 \pm 0.23$ & $10.07 \pm 0.23$ \\
\hline $\mathrm{Ce}_{2} \mathrm{O}_{3}$ & $9.55 \pm 0.22$ & 9.10 & \pm 0.22 & 9.17 & \pm 0.22 & $8.64 \pm 0.21$ & $8.45 \pm 0.20$ & $8.07 \pm 0.20$ \\
\hline $\mathrm{ThO}_{2}$ & $3.09 \pm 0.18$ & 3.42 & \pm 0.19 & 4.30 & \pm 0.20 & $8.38 \pm 0.27$ & $8.45 \pm 0.27$ & $8.21 \pm 0.27$ \\
\hline $\mathrm{Y}_{2} \mathrm{O}_{3}$ & $0.16 \pm 0.05$ & 0.00 & \pm 0.00 & 0.41 & \pm 0.06 & $0.03 \pm 0.04$ & $0.00 \pm 0.00$ & $0.11 \pm 0.04$ \\
\hline $\mathrm{Pr}_{2} \mathrm{O}_{3}$ & $0.27 \pm 0.07$ & 0.25 & \pm 0.07 & 0.39 & \pm 0.07 & $0.50 \pm 0.07$ & $0.36 \pm 0.07$ & $0.36 \pm 0.07$ \\
\hline $\mathrm{Nd}_{2} \mathrm{O}_{3}$ & $0.40 \pm 0.12$ & 0.31 & \pm 0.12 & 0.57 & \pm 0.12 & $0.31 \pm 0.12$ & $0.18 \pm 0.12$ & $0.38 \pm 0.12$ \\
\hline $\mathrm{CaO}$ & $0.69 \pm 0.03$ & 0.58 & \pm 0.03 & 0.68 & \pm 0.03 & $0.34 \pm 0.02$ & $0.27 \pm 0.02$ & $0.37 \pm 0.02$ \\
\hline $\mathrm{K}_{2} \mathrm{O}$ & $0.14 \pm 0.02$ & 0.11 & \pm 0.02 & 0.23 & \pm 0.02 & $0.26 \pm 0.02$ & $0.17 \pm 0.02$ & $0.17 \pm 0.02$ \\
\hline $\mathrm{Na}_{2} \mathrm{O} \#$ & 14.05 & 13.96 & & 13.85 & & 13.36 & 13.45 & 13.80 \\
\hline $\mathrm{F}$ & $0.22 \pm 0.02$ & 0.21 & \pm 0.02 & 0.15 & \pm 0.02 & $0.37 \pm 0.03$ & $0.30 \pm 0.03$ & $0.32 \pm 0.03$ \\
\hline $\mathrm{H}_{2} \mathrm{O}^{\#}$ & 5.48 & 5.44 & & 5.41 & & 5.25 & 5.26 & 5.39 \\
\hline $\mathrm{SO}_{3}$ & $0.54 \pm 0.06$ & 0.69 & \pm 0.06 & 0.87 & \pm 0.06 & $1.70 \pm 0.09$ & $0.97 \pm 0.07$ & $1.37 \pm 0.08$ \\
\hline Total & 100.61 & 99.11 & & 99.88 & & 100.35 & 100.06 & 101.57 \\
\hline $\mathrm{O}=\mathrm{F}$ & 0.09 & 0.09 & & 0.07 & & 0.16 & 0.13 & 0.13 \\
\hline Total & 100.52 & 99.02 & & 99.81 & & 100.19 & 99.93 & 101.44 \\
\hline $\mathrm{Na}_{2} \mathrm{O}_{\text {EMP }}$ & $5.78 \pm 0.26$ & 6.60 & \pm 0.28 & 7.39 & \pm 0.30 & $8.64 \pm 0.32$ & $7.93 \pm 0.31$ & $7.40 \pm 0.30$ \\
\hline $\mathrm{Si}$ & 5.96 & 5.94 & & 5.93 & & 5.85 & 5.92 & 5.89 \\
\hline $\mathrm{S}$ & 0.04 & 0.06 & & 0.07 & & 0.15 & 0.08 & 0.11 \\
\hline$\Sigma T$ & 6.00 & 6.00 & & 6.00 & & 6.00 & 6.00 & 6.00 \\
\hline $\mathrm{Na}_{\text {(calc) }}$ & 2.98 & 2.98 & & 2.97 & & 2.96 & 2.97 & 2.98 \\
\hline K & 0.02 & 0.02 & & 0.03 & & 0.04 & 0.03 & 0.02 \\
\hline$\Sigma$ Alc & 3.00 & 3.00 & & 3.00 & & 3.00 & 3.00 & 3.00 \\
\hline $\mathrm{Ca}$ & 0.08 & 0.07 & & 0.08 & & 0.04 & 0.03 & 0.04 \\
\hline $\mathrm{La}$ & 0.47 & 0.45 & & 0.42 & & 0.42 & 0.43 & 0.41 \\
\hline $\mathrm{Ce}$ & 0.38 & 0.37 & & 0.37 & & 0.36 & 0.35 & 0.33 \\
\hline $\operatorname{Pr}$ & 0.01 & 0.01 & & 0.02 & & 0.02 & 0.02 & 0.01 \\
\hline $\mathrm{Nd}$ & 0.02 & 0.01 & & 0.02 & & 0.01 & 0.01 & 0.02 \\
\hline Th & 0.08 & 0.09 & & 0.11 & & 0.22 & 0.22 & 0.21 \\
\hline Y & 0.01 & 0.00 & & 0.02 & & 0.00 & 0.00 & 0.01 \\
\hline$\Sigma M$ & 1.05 & 1.00 & & 1.04 & & 1.07 & 1.06 & 1.03 \\
\hline $\mathrm{F}$ & 0.08 & 0.07 & & 0.05 & & 0.13 & 0.11 & 0.11 \\
\hline $\mathrm{H}_{2} \mathrm{O}_{\text {(calc) }}$ & 2.00 & 2.00 & & 2.00 & & 2.00 & 2.00 & 2.00 \\
\hline ss Alc & 33.1 & 33.1 & & 33.3 & & 33.3 & 33.2 & 33.2 \\
\hline ss $M$ & 59.8 & 57.8 & & 60.1 & & 67.2 & 67.0 & 64.4 \\
\hline
\end{tabular}

ss $=$ calculated site-scatterings, in electrons per formula unit (e.p.f.u.)

\# calculated by stoichiometry in order to obtain 3 a.p.f.u. at the $\mathrm{Na}(1), \mathrm{Na}(2)$ and $\mathrm{Na}(3)$ sites and $2 \mathrm{H}_{2} \mathrm{O}$ p.f.u.

implying a more complicated substitution scheme involving other structural sites.

\section{Secondary-ion mass-spectrometry (SIMS)}

A Cameca IMS 4f ion microprobe was used in this study. Analytical conditions and procedures were described by Ottolini et al. (2004). SIMS data are reported in Table 2. The formula was calculated on the basis of $17(\mathrm{O}+\mathrm{F})$ per formula unit (p.f.u.), considering a stoichiometric quantity of $2 \mathrm{H}_{2} \mathrm{O}$ p.f.u. (corresponding to 5.51 wt.\%) from structure refinement data. The latter is higher than the value obtained by SIMS and reported in Table 2 (see chemical formula of sazhinite section for a discussion). Agreement for $R E E$ and Th between SIMS and EMPA for crystal 1 are within analytical error. In particular, the concentrations for $\mathrm{Pr}$ and $\mathrm{Nd}$ measured at the electron microprobe are close to the EMPA detection 
TABLE 1b. Electron-microprobe analyses and formulae on the basis of $\mathrm{Si}+\mathrm{S}=6$ a.p.f.u. for crystal 2.

\begin{tabular}{|c|c|c|c|c|c|c|c|c|}
\hline & 7 & 8 & 9 & 10 & 11 & 12 & 13 & 14 \\
\hline $\mathrm{SiO}_{2}$ & $53.97 \pm 1.43$ & $55.67 \pm 1.47$ & $53.71 \pm 1.43$ & $53.47 \pm 1.42$ & $52.14 \pm 1.39$ & $51.94 \pm 1.38$ & $52.79 \pm 1.40$ & $53.12 \pm 1.41$ \\
\hline $\mathrm{La}_{2} \mathrm{O}_{3}$ & $12.44 \pm 0.26$ & $12.52 \pm 0.26$ & $9.70 \pm 0.22$ & $8.43 \pm 0.20$ & $9.69 \pm 0.22$ & $8.66 \pm 0.20$ & $9.05 \pm 0.21$ & $9.10 \pm 0.21$ \\
\hline $\mathrm{Ce}_{2} \mathrm{O}_{3}$ & $10.89 \pm 0.24$ & $11.52 \pm 0.25$ & $10.61 \pm 0.24$ & $11.34 \pm 0.25$ & $11.53 \pm 0.25$ & $9.98 \pm 0.23$ & $9.84 \pm 0.23$ & $10.10 \pm 0.23$ \\
\hline $\mathrm{ThO}_{2}$ & $0.76 \pm 0.11$ & $0.75 \pm 0.12$ & $5.39 \pm 0.22$ & $5.33 \pm 0.22$ & $5.79 \pm 0.23$ & $4.81 \pm 0.21$ & $3.66 \pm 0.19$ & $3.71 \pm 0.19$ \\
\hline $\mathrm{Y}_{2} \mathrm{O}_{3}$ & $0.00 \pm 0.00$ & $0.00 \pm 0.00$ & $0.00 \pm 0.00$ & $0.07 \pm 0.05$ & $0.14 \pm 0.04$ & $0.86 \pm 0.07$ & $0.67 \pm 0.08$ & $1.02 \pm 0.08$ \\
\hline $\mathrm{Pr}_{2} \mathrm{O}_{3}$ & $0.46 \pm 0.07$ & $0.32 \pm 0.07$ & $0.45 \pm 0.07$ & $0.48 \pm 0.07$ & $0.37 \pm 0.07$ & $0.54 \pm 0.07$ & $0.40 \pm 0.07$ & $0.46 \pm 0.07$ \\
\hline $\mathrm{Nd}_{2} \mathrm{O}_{3}$ & $0.00 \pm 0.00$ & $0.09 \pm 0.12$ & $0.62 \pm 0.12$ & $0.92 \pm 0.12$ & $0.37 \pm 0.12$ & $0.82 \pm 0.12$ & $0.72 \pm 0.12$ & $1.05 \pm 0.13$ \\
\hline $\mathrm{CaO}$ & $0.40 \pm 0.02$ & $0.34 \pm 0.02$ & $0.18 \pm 0.02$ & $0.19 \pm 0.02$ & $0.04 \pm 0.02$ & $0.27 \pm 0.02$ & $0.25 \pm 0.02$ & $0.27 \pm 0.02$ \\
\hline $\mathrm{K}_{2} \mathrm{O}$ & $0.12 \pm 0.02$ & $0.09 \pm 0.02$ & $0.28 \pm 0.02$ & $0.27 \pm 0.02$ & $0.21 \pm 0.02$ & $0.34 \pm 0.02$ & $0.26 \pm 0.02$ & $0.33 \pm 0.02$ \\
\hline $\mathrm{Na}_{2} \mathrm{O}^{\#}$ & 13.85 & 14.32 & 13.67 & 13.62 & 13.52 & 13.19 & 13.45 & 13.47 \\
\hline $\mathrm{F}$ & $0.12 \pm 0.02$ & $0.11 \pm 0.02$ & $0.11 \pm 0.02$ & $0.12 \pm 0.02$ & $0.22 \pm 0.02$ & $0.12 \pm 0.02$ & $0.10 \pm 0.02$ & $0.15 \pm 0.02$ \\
\hline $\mathrm{H}_{2} \mathrm{O}^{\#}$ & 5.39 & 5.56 & 5.37 & 5.34 & 5.29 & 5.19 & 5.27 & 5.31 \\
\hline $\mathrm{SO}_{3}$ & $0.00 \pm 0.00$ & $0.00 \pm 0.00$ & $0.04 \pm 0.03$ & $0.01 \pm 0.04$ & $1.12 \pm 0.07$ & $0.02 \pm 0.04$ & $0.03 \pm 0.03$ & $0.02 \pm 0.04$ \\
\hline Total & 98.40 & 101.29 & 100.13 & 99.59 & 100.43 & 96.74 & 96.49 & 98.11 \\
\hline $\mathrm{O}=\mathrm{F}$ & 0.05 & 0.05 & 0.05 & 0.05 & 0.09 & 0.05 & 0.04 & 0.06 \\
\hline Total & 99.35 & 101.24 & 100.08 & 99.54 & 100.34 & 96.69 & 96.45 & 98.05 \\
\hline $\mathrm{Na}_{2} \mathrm{O}$ & $12.35 \pm 0.38$ & $11.03 \pm 0.36$ & $10.45 \pm 0.35$ & $10.60 \pm 0.36$ & $11.89 \pm 0.38$ & $10.67 \pm 0.35$ & $10.44 \pm 0.35$ & $10.96 \pm 0.36$ \\
\hline $\mathrm{Si}$ & 6.00 & 6.00 & 6.00 & 6.00 & 5.91 & 6.00 & 6.00 & 6.00 \\
\hline S & & & & & 0.09 & & & \\
\hline$\Sigma T$ & 6.00 & 6.00 & 6.00 & 6.00 & 6.00 & 6.00 & 6.00 & 6.00 \\
\hline $\mathrm{Na}_{\text {(calc) }}$ & 2.98 & 2.99 & 2.96 & 2.96 & 2.97 & 2.95 & 2.96 & 2.95 \\
\hline $\mathrm{K}$ & 0.02 & 0.01 & 0.04 & 0.04 & 0.03 & 0.05 & 0.04 & 0.05 \\
\hline$\Sigma$ Alc & 3.00 & 3.00 & 3.00 & 3.00 & 3.00 & 3.00 & 3.00 & 3.00 \\
\hline $\mathrm{Ca}$ & 0.05 & 0.04 & 0.02 & 0.02 & 0.00 & 0.03 & 0.03 & 0.03 \\
\hline $\mathrm{La}$ & 0.51 & 0.50 & 0.40 & 0.35 & 0.41 & 0.37 & 0.38 & 0.38 \\
\hline $\mathrm{Ce}$ & 0.44 & 0.46 & 0.43 & 0.47 & 0.48 & 0.42 & 0.41 & 0.42 \\
\hline $\operatorname{Pr}$ & 0.02 & 0.01 & 0.02 & 0.02 & 0.02 & 0.02 & 0.02 & 0.02 \\
\hline $\mathrm{Nd}$ & 0.00 & 0.00 & 0.03 & 0.04 & 0.02 & 0.03 & 0.03 & 0.04 \\
\hline Th & 0.02 & 0.02 & 0.14 & 0.17 & 0.15 & 0.13 & 0.10 & 0.10 \\
\hline $\mathrm{Y}$ & 0.00 & 0.00 & 0.00 & 0.00 & 0.01 & 0.05 & 0.04 & 0.06 \\
\hline$\Sigma M$ & 1.04 & 1.03 & 1.04 & 1.07 & 1.09 & 1.05 & 1.01 & 1.05 \\
\hline $\mathrm{F}$ & 0.04 & 0.04 & 0.04 & 0.04 & 0.08 & 0.04 & 0.04 & 0.05 \\
\hline $\mathrm{H}_{2} \mathrm{O}_{\text {(calc) }}$ & 2.00 & 2.00 & 2.00 & 2.00 & 2.00 & 2.00 & 2.00 & 2.00 \\
\hline ss $\mathrm{Na}$ & 33.2 & 33.1 & 33.3 & 33.3 & 33.2 & 33.4 & 33.3 & 33.4 \\
\hline ss $M$ & 58.6 & 58.4 & 63.7 & 66.5 & 67.5 & 62.7 & 59.6 & 61.5 \\
\hline
\end{tabular}

ss $=$ calculated site-scatterings, in e.p.f.u.

\# calculated by stoichiometry in order to obtain 3 a.p.f.u. at $\mathrm{Na}(1), \mathrm{Na}(2)$ and $\mathrm{Na}(3)$ sites and $2 \mathrm{H}_{2} \mathrm{O}$ p.f.u.

limits for these two elements. However, the $\mathrm{Na}_{2} \mathrm{O}$ content measured by EMPA is lower than the SIMS measurements, the latter in good agreement with the $\mathrm{Na}$ content obtained by X-ray singlecrystal diffraction (see next section).

\section{X-ray crystallography}

A crystal was examined at CNR-IGG using a Bruker AXS SMART Apex single-crystal diffractometer with a $5 \mathrm{~cm}$ crystal-to-detector distance and graphite-monochromatized Mo- $K \alpha$ X-radiation operating at $40 \mathrm{kV}$ and $40 \mathrm{nA}$. Three-dimensional data were integrated and corrected for Lorentz, polarization, and background effects using the SAINT+ software version 6.02 (® B Bruker AXS). Unit-cell dimensions reported (Table 3) were calculated from the least-squares refinement of the positions of all the collected reflections. Frame widths of $0.2^{\circ}$ in $\omega$ were used to collect 900 frames per batch in three batches at different $\phi$ values $\left(0^{\circ}\right.$, $\left.120^{\circ}, 240^{\circ}\right)$. Counting time per image was $20 \mathrm{~s}$. Raw intensity data were corrected for absorption using SADABS v. 2.03 program (Sheldrick, 1996). 

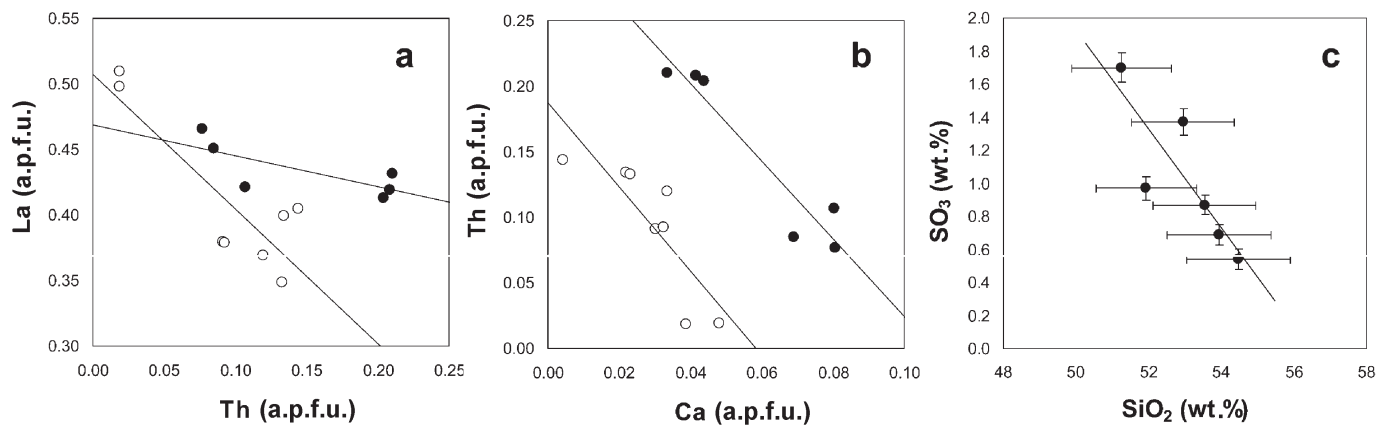

Fig. 4. Correlation of (a) Th vs. Ca, (b) La vs. Th (c) $\mathrm{SO}_{3} v s$. $\mathrm{SiO}_{2}$ in wt.\%. Filled dots are for crystal 1 and open dots for crystal 2 .

TABLE 2. Results of SIMS and EMP analysis and formulae on the basis of $17(\mathrm{O}+\mathrm{F})$.

\begin{tabular}{|c|c|c|c|}
\hline Point & A & & A \\
\hline $\mathrm{SiO}_{2} *$ & 53.99 & $\mathrm{Si}$ & 5.87 \\
\hline $\mathrm{La}_{2} \mathrm{O}_{3}$ & 10.18 & $\mathrm{~S}$ & 0.06 \\
\hline $\mathrm{Ce}_{2} \mathrm{O}_{3}$ & 8.68 & B & 0.01 \\
\hline $\mathrm{ThO}_{2}$ & 3.63 & $\Sigma \mathrm{Si}$ sites & 5.94 \\
\hline $\mathrm{UO}_{2}$ & 0.22 & $\mathrm{Sr}$ & 0.01 \\
\hline $\mathrm{ZrO}_{2}$ & 0.18 & $\mathrm{Na}$ & 2.87 \\
\hline $\mathrm{Y}_{2} \mathrm{O}_{3}$ & 0.25 & $\mathrm{~K}$ & 0.02 \\
\hline $\mathrm{Pr}_{2} \mathrm{O}_{3}$ & 0.56 & $\mathrm{Ba}$ & 0.00 \\
\hline $\mathrm{Nd}_{2} \mathrm{O}_{3}$ & 1.08 & $\Sigma \mathrm{Na}$ sites & 2.90 \\
\hline $\mathrm{Sm}_{2} \mathrm{O}_{3}$ & 0.08 & $\mathrm{La}$ & 0.41 \\
\hline $\mathrm{Eu}_{2} \mathrm{O}_{3}$ & 0.01 & $\mathrm{Ce}$ & 0.35 \\
\hline $\mathrm{Gd}_{2} \mathrm{O}_{3}$ & 0.04 & $\operatorname{Pr}$ & 0.02 \\
\hline $\mathrm{Dy}_{2} \mathrm{O}_{3}$ & 0.04 & $\mathrm{Nd}$ & 0.04 \\
\hline $\mathrm{Er}_{2} \mathrm{O}_{3}$ & 0.03 & $\mathrm{Sm}$ & 0.003 \\
\hline $\mathrm{Yb}_{2} \mathrm{O}_{3}$ & 0.03 & $\mathrm{Eu}$ & 0.000 \\
\hline $\mathrm{CaO}^{*}$ & 0.65 & $\mathrm{Gd}$ & 0.001 \\
\hline $\mathrm{SrO}$ & 0.15 & Dy & 0.001 \\
\hline $\mathrm{BaO}$ & 0.01 & Er & 0.001 \\
\hline $\mathrm{Li}_{2} \mathrm{O}$ & 0.02 & $\mathrm{Yb}$ & 0.001 \\
\hline $\mathrm{Na}_{2} \mathrm{O}$ & 13.60 & Y & 0.01 \\
\hline $\mathrm{F}$ & 0.41 & $\mathrm{Zr}$ & 0.01 \\
\hline $\mathrm{K}_{2} \mathrm{O}^{*}$ & 0.16 & Th & 0.09 \\
\hline $\mathrm{B}_{2} \mathrm{O}_{3}$ & 0.04 & $\mathrm{U}$ & 0.01 \\
\hline $\mathrm{H}_{2} \mathrm{O}^{\#}$ & 5.51 & $\mathrm{Li}$ & 0.01 \\
\hline $\mathrm{SO}_{3} *$ & 0.70 & $\mathrm{Ca}$ & 0.08 \\
\hline Total & 100.25 & $\Sigma M$ & 1.04 \\
\hline $\mathrm{O}=\mathrm{F}$ & 0.17 & F & 0.14 \\
\hline \multirow[t]{2}{*}{ Total } & 100.08 & $\mathrm{H}_{2} \mathrm{O}$ & 2.00 \\
\hline & & $\operatorname{ss} M$ & 59.1 \\
\hline $\mathrm{H}_{2} \mathrm{O}_{\text {SIMS }}$ & 1.79 & ss $\Sigma \mathrm{Na}$ & 32.3 \\
\hline
\end{tabular}

ss $=$ site scattering calculated in electrons per formula unit (e.p.f.u.)

* Averages of EMPA data close to SIMS spots

\# stoichiometric $\mathrm{H}_{2} \mathrm{O}$ content to obtain $2 \mathrm{H}_{2} \mathrm{O}$ p.f.u.

The structure was refined with the software package SHELXTL version 6.10 (®) Bruker AXS). Starting atom coordinates were taken from Shumyatskaya et al. (1980). DifferenceFourier maps showed the presence of two residua near $4 \mathrm{e}^{-} / \AA^{3}$ that were added to the model as $\mathrm{Na}(3)$ and $\mathrm{O}(14)$. Refinement of occupancies of these two sites $\mathrm{Na}^{+}$and $\mathrm{O}$ scattering curves, respectively) yielded half-occupancy factors. Final refined atom coordinates and atom-displace-

TABLE 3. Crystal data and structure refinement for the crystal studied.

IGG identification code

iax

Wavelength ( $(\AA)$

Crystal system

Space group

Unit-cell dimensions (

Volume $\left(\AA^{3}\right)$

Z

Absorption coefficient $\left(\mathrm{mm}^{-1}\right)$

$\theta$ range for data collection $\left({ }^{\circ}\right)$

Index ranges

Reflections collected

Independent reflections

$R$ (int)

Completeness to $\theta=33.66^{\circ}$

Data/restraints/parameters

Goodness-of-fit on $F^{2}$

Final $R$ indices $[\mathrm{I}>2 \sigma(\mathrm{I})]$

$R$ indices (all data)
0.71073

Orthorhombic

Pmm2

$a=7.415(2)$

$b=15.515(3)$

$c=7.164(1)$

$824.2(3)$

2

0.653

2.6 to 33.7

$-11 \leqslant h \leqslant 11$,

$-23 \leqslant k \leqslant 23$,

$-11 \leqslant l \leqslant 10$

10,072

3369

0.025

$96.9 \%$

$3369 / 2 / 150$

1.146

$R 1=0.037$

$w R 2=0.092$

$R 1=0.038$

$w R 2=0.093$ 
SAZHINITE-(LA) FROM ARIS

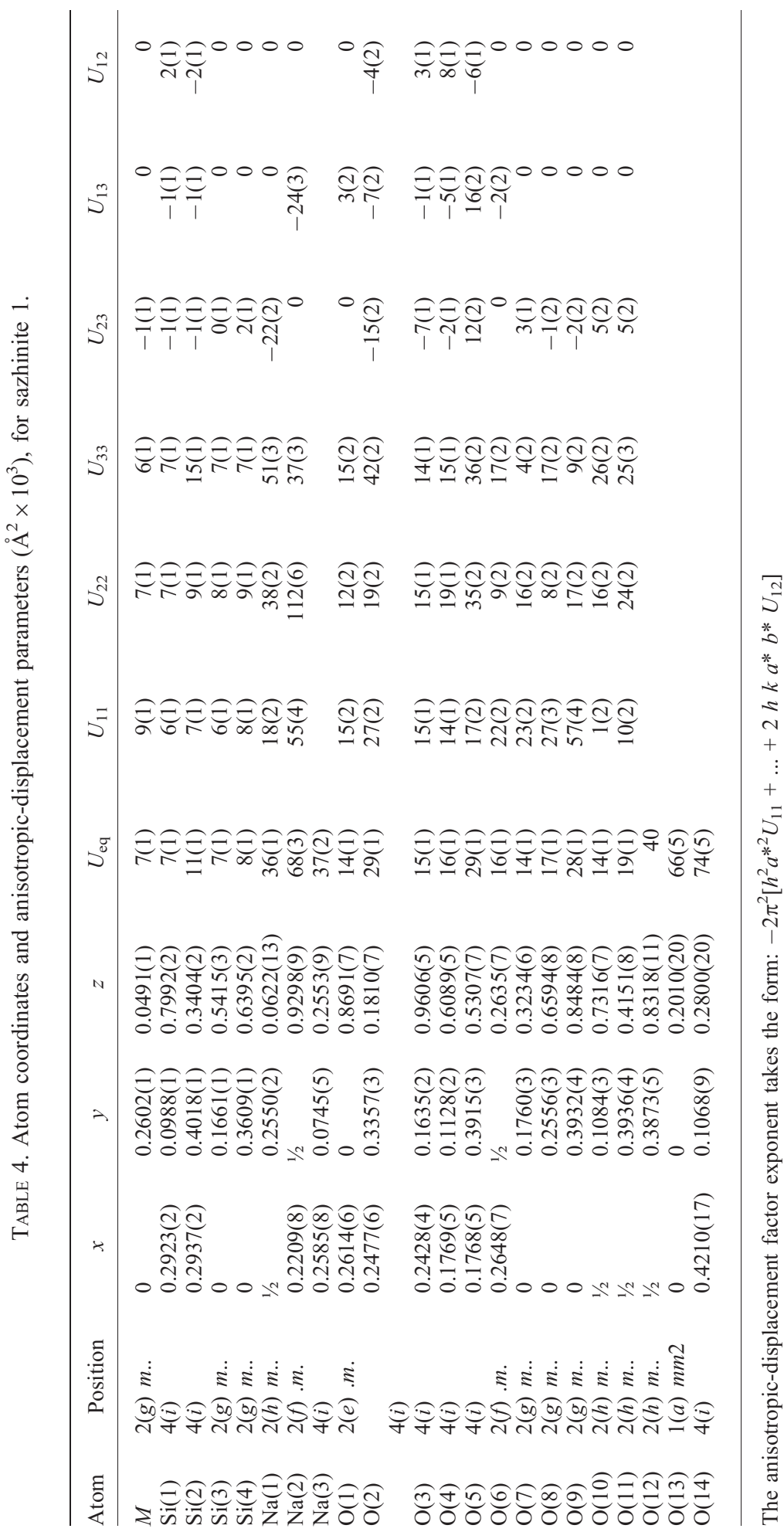


ment parameters are reported in Table 4. Selected interatomic distances are reported in Table 5. Observed and calculated structure factors are reported in Table 6 (deposited with the editor and available from www.minersoc.org/e journals/ dep_mat.html). Due to extensive intra-crystalline chemical zoning, the X-ray powder-diffraction pattern (Table 7) was calculated from the refined structure model.

\section{Description of the structure}

Shumyatskaya et al. (1980) described sazhinite$(\mathrm{Ce})$ as a member of the dalyite family. Dalyite $\left(\mathrm{K}_{2} \mathrm{ZrSi}_{6} \mathrm{O}_{15}\right.$, Fleet, 1965), sazhinite-(Ce), and sazhinite-(La) have structures comprising wavy layers of silica tetrahedra condensed to xonotlitelike chains, and forming alternating four-, six- and eight-membered rings (Fig. 5a). Tetrahedra share three of their four apices with neighbouring tetrahedra. Xonotlite-like chains are formed by condensation of wollastonite-like chains, in which two tetrahedra point downward and one tetrahedron points upward. In sazhinite the corrugated layers are linked by [7]-coordinated REE and minor quantities of actinides at the $M$ site
(Fig. 5b), which can be described as a capped octahedron. The silica-REE framework gives rise to channels that contain three alkali cations p.f.u., compensating for the residual charge in the corrugated layers. The $\left[\mathrm{Si}_{6} \mathrm{O}_{15}\right]^{6-}$ sheets are topologically equivalent in sazhinite and in the synthetic compound $\beta-\mathrm{K}_{3} \mathrm{NdSi}_{6} \mathrm{O}_{15}$ (Haile and Wuensch, 2000); however, the positions of cations in the channels are different. Small cations $(\mathrm{Na})$ coordinate $\mathrm{H}_{2} \mathrm{O}$ groups, whereas large cations $(\mathrm{K})$ occupy the position of the $\mathrm{H}_{2} \mathrm{O}$ group in sazhinite.

The local structure around $\mathrm{Na}$ sites is shown in Fig. 6. From Fig. $6 a$, it is evident that the $\mathrm{Na}(1)$ atom can be 5-fold or 6-fold coordinated depending on whether the $\mathrm{O}(14)$ anion is vacant or not. A vacancy at the $\mathrm{O}(14)$ site does not encompass a larger impact on the bond valence incidence at the $\mathrm{Na}(1)$ site [see bond-valence table for sazhinite-(La) at Table 8]. Positional disorder at the $\mathrm{O}(14)$ anion site is probably related to the oblate ellipsoid of the $\mathrm{Na}(1)$ site. The $\mathrm{Na}(2)$ atom is always 5-fold coordinated and the large thermal displacement along the $b$ axis may be related to high thermal motion of $\mathrm{H}_{2} \mathrm{O}$ groups at the $\mathrm{O}(12)$ site. Inspection of Table 8 , also shows a smaller

TABLE 5. Selected interatomic distances $(\AA)$.

\begin{tabular}{llll}
\hline $\mathrm{Si}(1)-\mathrm{O}(3)$ & $1.575(3)$ & $\mathrm{Si}(2)-\mathrm{O}(2)$ & $1.572(4)$ \\
$\mathrm{Si}(1)-\mathrm{O}(10)$ & $1.621(2)$ & $\mathrm{Si}(2)-\mathrm{O}(5)$ & $1.624(5)$ \\
$\mathrm{Si}(1)-\mathrm{O}(4)$ & $1.624(3)$ & $\mathrm{Si}(2)-\mathrm{O}(11)$ & $1.626(2)$ \\
$\mathrm{Si}(1)-\mathrm{O}(1)$ & $1.629(2)$ & $\mathrm{Si}(2)-\mathrm{O}(6)$ & $1.634(2)$ \\
$<\mathrm{Si}(1)-\mathrm{O}>$ & 1.612 & $<\mathrm{Si}(2)-\mathrm{O}>$ & 1.614 \\
$\mathrm{Si}(3)-\mathrm{O}(7)$ & $1.570(5)$ & $\mathrm{Si}(4)-\mathrm{O}(9)$ & $1.578(6)$ \\
$\mathrm{Si}(3)-\mathrm{O}(4) \times 2$ & $1.624(3)$ & $\mathrm{Si}(4)-\mathrm{O}(5) \times 2$ & $1.597(4)$ \\
$\mathrm{Si}(3)-\mathrm{O}(8)$ & $1.625(5)$ & $\mathrm{Si}(4)-\mathrm{O}(8)$ & $1.640(5)$ \\
$<\mathrm{Si}(3)-\mathrm{O}>$ & 1.611 & $<\mathrm{Si}(4)-\mathrm{O}>$ & 1.603 \\
$M-\mathrm{O}(7)$ & $2.360(4)$ & $\mathrm{Na}(1)-\mathrm{O}(2) \times 2$ & $2.407(5)$ \\
$M-\mathrm{O}(2) \times 2$ & $2.375(4)$ & $\mathrm{Na}(1)-\mathrm{O}(3)^{\# 2} \times 2$ & $2.486(4)$ \\
$M-\mathrm{O}(3)^{\# 1} \times 2$ & $2.428(3)$ & $\mathrm{Na}(1)-\mathrm{O}(12)^{\# 2}$ & $2.634(11)$ \\
$M-\mathrm{O}(9)^{\# 2}$ & $2.515(6)$ & $\mathrm{Na}(1)-\mathrm{O}(14)^{\# 4}$ & $2.840(16)$ \\
$M-\mathrm{O}(8)^{\# 2}$ & $2.793(6)$ & $<\mathrm{Na}(1)-\mathrm{O}>$ & 2.543 \\
$<M-\mathrm{O}>$ & 2.468 & & \\
& & $\mathrm{Na}(2)-\mathrm{O}(9) \times 2$ & $2.402(6)$ \\
$\mathrm{Na}(3)-\mathrm{O}(13)^{\# 7}$ & $2.272(7)$ & $\mathrm{Na}(2)-\mathrm{O}(6)^{\# 5}$ & $2.413(8)$ \\
$\mathrm{Na}(3)-\mathrm{O}(14)^{\# 6}$ & $2.435(15)$ & $\mathrm{Na}(2)-\mathrm{O}(12) \times 2$ & $2.799(7)$ \\
$\mathrm{Na}(3)-\mathrm{O}(3)^{\# 8}$ & $2.526(7)$ & $<\mathrm{Na}(2)-\mathrm{O}>$ & 2.563 \\
$\mathrm{Na}(3)-\mathrm{O}(7)^{\# 4}$ & $2.528(7)$ & & \\
$\mathrm{Na}(3)-\mathrm{O}(4)^{\# 4}$ & $2.672(8)$ & $\mathrm{Na}(2)-\mathrm{Na}(2)^{\# 3}$ & $3.276(12)$ \\
$<\mathrm{Na}(3)-\mathrm{O}>$ & 2.487 & $\mathrm{Na}(3)-\mathrm{Na}(1)^{\# 4}$ & $3.600(8)$ \\
\hline
\end{tabular}

Symmetry transformations used to generate equivalent atoms:

$\# 1-x, y, z-1 \quad \# 2 x, y, z-1 \quad \# 3-x,-y+1, z$ $\# 4 x,-y+1, z \quad \# 5 x, y, z+1 \quad \# 6-x+1, y, z$ 
TABLE 7. Calculated powder-diffraction pattern of sazhinite-(La) $\left(I / I_{\mathrm{o}} \geqslant 5\right.$; the nine strongest reflections are in bold).

\begin{tabular}{|c|c|c|c|c|c|c|}
\hline$I / I_{\mathrm{o}}$ & ${ }^{\circ} 2 \theta$ & $d_{\text {(calc) }}(\AA)$ & no.* & $h$ & $k$ & $l$ \\
\hline 17.8 & 11.41 & 7.758 & & 0 & 2 & 0 \\
\hline 59.1 & 11.94 & 7.415 & & 1 & 0 & 0 \\
\hline 16.3 & 12.35 & 7.164 & 2 & 0 & 0 & 1 \\
\hline 47.6 & 13.61 & 6.504 & 2 & 0 & 1 & 1 \\
\hline 59.9 & 16.54 & 5.360 & & 1 & 2 & 0 \\
\hline 68.0 & 16.84 & 5.263 & 2 & 0 & 2 & 1 \\
\hline 12.0 & 18.14 & 4.890 & 2 & 1 & 1 & 1 \\
\hline 11.2 & 20.70 & 4.292 & 2 & 1 & 2 & 1 \\
\hline 16.5 & 25.92 & 3.437 & & 1 & 4 & 0 \\
\hline 100.0 & 26.12 & 3.411 & 2 & 0 & 4 & 1 \\
\hline 45.4 & 26.65 & 3.345 & & 2 & 2 & 0 \\
\hline 83.0 & 27.42 & 3.252 & 2 & 0 & 2 & 2 \\
\hline 51.2 & 27.66 & 3.226 & 2 & 1 & 0 & 2 \\
\hline 44.9 & 27.70 & 3.221 & 2 & 2 & 1 & 1 \\
\hline 22.8 & 28.81 & 3.099 & 2 & 1 & 4 & 1 \\
\hline 12.7 & 29.47 & 3.031 & 2 & 2 & 2 & 1 \\
\hline 9.9 & 30.00 & 2.978 & 2 & 1 & 2 & 2 \\
\hline 11.5 & 31.42 & 2.847 & 2 & 0 & 5 & 1 \\
\hline 8.8 & 32.23 & 2.778 & 2 & 2 & 3 & 1 \\
\hline 11.8 & 33.43 & 2.680 & & 2 & 4 & 0 \\
\hline 21.9 & 34.07 & 2.632 & 2 & 0 & 4 & 2 \\
\hline 6.6 & 34.69 & 2.586 & & 0 & 6 & 0 \\
\hline 17.7 & 34.83 & 2.576 & 2 & 2 & 0 & 2 \\
\hline 14.8 & 35.77 & 2.510 & 2 & 2 & 4 & 1 \\
\hline 6.6 & 36.76 & 2.445 & 2 & 2 & 2 & 2 \\
\hline 7.5 & 38.38 & 2.345 & 2 & 0 & 5 & 2 \\
\hline 7.9 & 38.97 & 2.311 & 2 & 1 & 6 & 1 \\
\hline 16.6 & 39.48 & 2.282 & 2 & 0 & 2 & 3 \\
\hline 6.7 & 41.39 & 2.181 & 2 & 1 & 2 & 3 \\
\hline 9.1 & 42.11 & 2.146 & 2 & 2 & 4 & 2 \\
\hline 20.0 & 44.55 & 2.034 & 2 & 2 & 6 & 1 \\
\hline 6.0 & 44.55 & 2.034 & 2 & 0 & 4 & 3 \\
\hline 5.0 & 44.93 & 2.018 & 2 & 1 & 6 & 2 \\
\hline 7.6 & 45.16 & 2.008 & 2 & 2 & 0 & 3 \\
\hline 8.6 & 46.13 & 1.968 & 2 & 3 & 2 & 2 \\
\hline 9.4 & 46.29 & 1.961 & 2 & 1 & 4 & 3 \\
\hline 6.0 & 46.74 & 1.944 & 2 & 2 & 2 & 3 \\
\hline 6.0 & 46.84 & 1.939 & & 0 & 8 & 0 \\
\hline 11.6 & 49.15 & 1.854 & & 4 & 0 & 0 \\
\hline 6.5 & 49.97 & 1.825 & 2 & 2 & 6 & 2 \\
\hline 8.7 & 50.67 & 1.802 & 3 & 3 & 4 & 2 \\
\hline 6.1 & 50.99 & 1.791 & 2 & 0 & 0 & 4 \\
\hline 6.1 & 51.24 & 1.783 & 2 & 2 & 4 & 3 \\
\hline 10.2 & 52.13 & 1.754 & 2 & 0 & 6 & 3 \\
\hline 5.0 & 52.81 & 1.734 & 2 & 3 & 6 & 1 \\
\hline 6.8 & 57.20 & 1.610 & 2 & 4 & 2 & 2 \\
\hline 5.2 & 58.45 & 1.579 & 2 & 2 & 2 & 4 \\
\hline 5.3 & 63.14 & 1.472 & 2 & 0 & 6 & 4 \\
\hline 7.0 & 65.57 & 1.424 & 2 & 0 & 10 & 2 \\
\hline
\end{tabular}

$*$ no. $=$ number of overlapping reflections with $(h k \bar{l})$. bond valence incident sum for $\mathrm{Na}(2)$ that may be related to its high dynamic disorder. The different possible local configurations for the $\mathrm{Na}(3)$ site are shown in Fig. 6b: configuration 1 is not allowed because of very short $\mathrm{Na}(3)-\mathrm{Na}(3)$ distances $(2.31 \AA)$; all the other cases are possible. Configuration 2 is the one chosen for Fig. $5 a$.

\section{The chemical formula of sazhinite}

The $T$ sites are occupied by $\mathrm{Si}$ (with minor $\mathrm{S}$ ), and the $\mathrm{O}(1)-\mathrm{O}(11)$ sites are fully occupied by $\mathrm{O}^{2-}$ to give the structural unit $\left[\mathrm{Si}_{6} \mathrm{O}_{15}\right]^{6-}$ for $\mathrm{Z}=2$. The refined site scattering at the $M$ site (Table 2) is in accordance with REE occupancy, the dominant cation being $\mathrm{La}^{3+}$ (Table 2). The $\mathrm{Na}(1)$ and $\mathrm{Na}(2)$ sites are fully occupied and the $\mathrm{Na}(3)$ site is halfoccupied, resulting in an $\mathrm{Na}$ content of 3 a.p.f.u. (for $Z=2$ ), in accordance with the amount of $\mathrm{Na}$ determined by SIMS (Table 2). Inspection of the bond-valence table for sazhinite-(La) (Table 8) shows the $\mathrm{O}(12), \mathrm{O}(13)$ and $\mathrm{O}(14)$ sites to be occupied by $\mathrm{H}_{2} \mathrm{O}$, and hence we may write the $\mathrm{S}$ free formula as $\mathrm{Na}_{3} \mathrm{La}\left[\mathrm{Si}_{6} \mathrm{O}_{15}\right]\left(\mathrm{H}_{2} \mathrm{O}\right)_{n}$. What is the value of $n$ ? Inspection of Table 8 shows that $\mathrm{O}(13)$ and $\mathrm{O}(14)$ are close to $\mathrm{Na}(3)$; if $\mathrm{Na}(3)$ is unoccupied, $\mathrm{O}(13)$ and $\mathrm{O}(14)$ have no significant role in the structure and are probably unoccupied, giving an $\mathrm{H}_{2} \mathrm{O}$ content of 1 p.f.u., corresponding to the $\mathrm{H}_{2} \mathrm{O}$ group at the $\mathrm{O}(12)$ site. If $\mathrm{Na}(3)$ is halfoccupied then $\mathrm{O}(14)$ should be half-occupied. The refinement gives $\mathrm{O}(14)$ as half-occupied and it is probable that the high displacement factor of this anion site (Table 4) indicates further partial occupancy down to 0.25 , thus summing up $2 \mathrm{H}_{2} \mathrm{O}$ p.f.u. Note that Ottolini et al. (2004) give a much lower $\mathrm{H}_{2} \mathrm{O}$ content, and ascribe the difference to loss of weakly-bound $\mathrm{H}_{2} \mathrm{O}$ molecules in the crystal structure under the high vacuum of the ion microprobe during SIMS analysis.

\section{Chemical substitutions in sazhinite-(La)}

The end-member formula for sazhinite-( $\mathrm{La})$ is $\mathrm{Na}_{3} \mathrm{La}\left[\mathrm{Si}_{6} \mathrm{O}_{15}\right]\left(\mathrm{H}_{2} \mathrm{O}\right)_{2}$ and the principal substituents in this structure are (in order of decreasing importance and ignoring homovalent substitutions) $\mathrm{Th}^{4+}, \mathrm{S}^{6+}$ and $\mathrm{Ca}^{2+}$. These atoms have valences different from the cation species in the end-member compositions, and hence their incorporation must be as coupled with heterovalent substitutions. For example, $\mathrm{Th}^{4+}$ substitution for $R E E^{3+}$ at the $M$ site must accompany the substitution of a lower-valence species. Hence $S^{6+}$ 

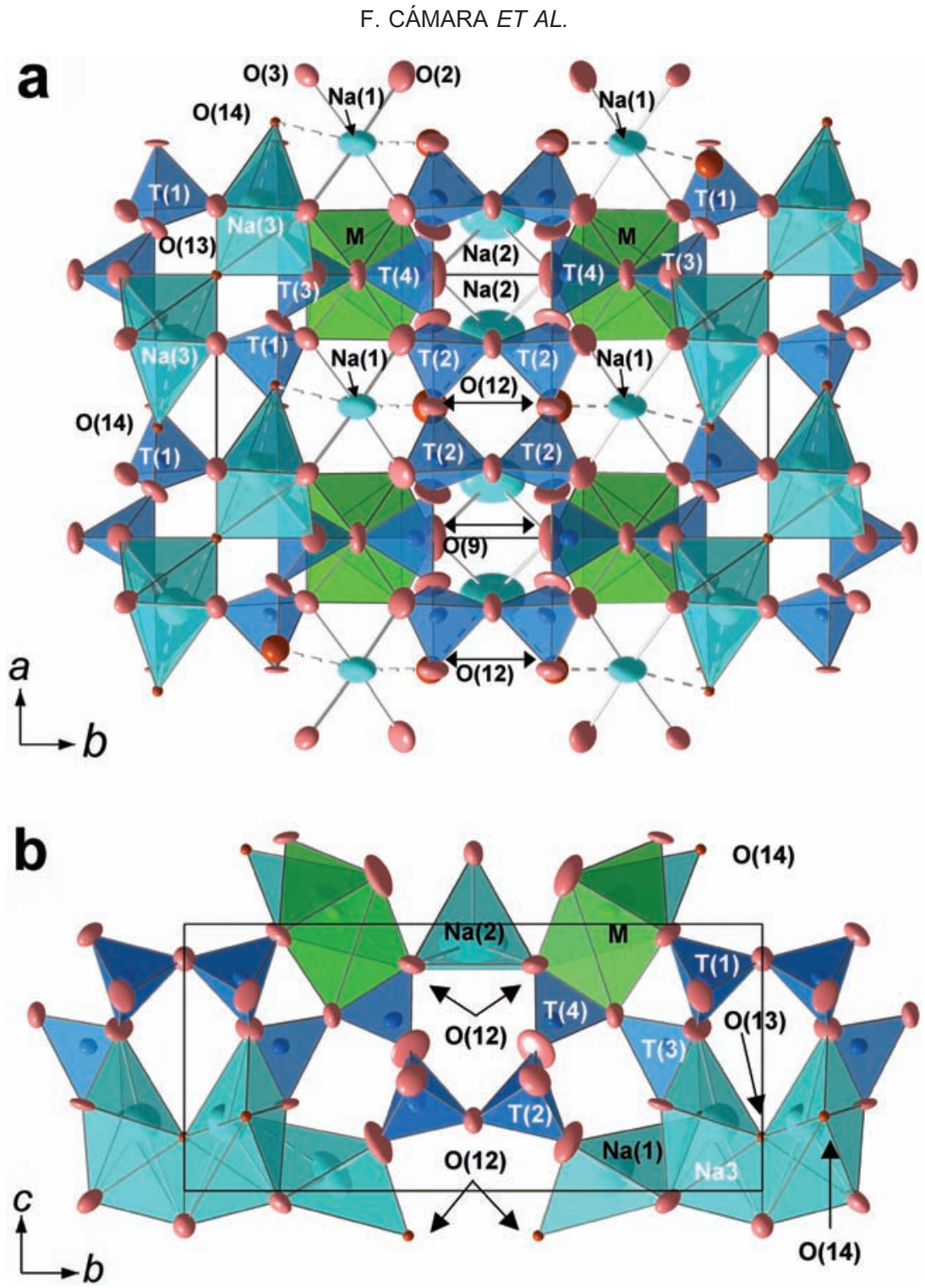

FIG. 5. Projection of the sazhinite structure $(a)$ onto $(001)$ and $(b)$ onto (100). In $(a)$ the $\mathrm{Na}(1)$ and $\mathrm{Na}(2)$ sites and coordinating $\mathrm{O}$ atoms are shown as ellipsoids at $95 \%$ probability. In both figures the $\mathrm{O}(12), \mathrm{O}(13)$ and $\mathrm{O}(14) \mathrm{O}$ atoms are shown as spheres.

cannot be involved in this substitution. In principle, $\mathrm{Th}^{4+} \rightarrow R E E^{3+}$ could be accompanied by $\mathrm{Ca}^{2+} \rightarrow R E E^{3+}$, but this substitution does not occur since $\mathrm{Th}^{4+}$ and $\mathrm{Ca}^{2+}$ are negatively correlated (Fig. $4 b$ ). The likely substitution that compensates the excess charge produced by $\mathrm{Th}^{4+}$ $\rightarrow R E E^{3+}$ is $\square \rightarrow \mathrm{Na}$, and hence $\mathrm{Th}^{4+}$ is incorporated into the sazhinite-(La) via the substitution $\mathrm{Th}^{4+}+\square \rightarrow R E E^{3+}+\mathrm{Na}$.
We cannot measure Na accurately by EMPA in sazhinite-(La) and hence cannot check the variation in Na. However, this is a likely way in which the additional charge of $\mathrm{Th}^{4+}$ can be accounted for, since substitution for Si produces unacceptable local bond-valence sums. In addition, the $\mathrm{Na}(3)$ site shares two $\mathrm{O}(3)$ and one $\mathrm{O}(7)$ anions with the $M$ site. Thus the entrance of highcharge cations at the $M$ site would compensate 

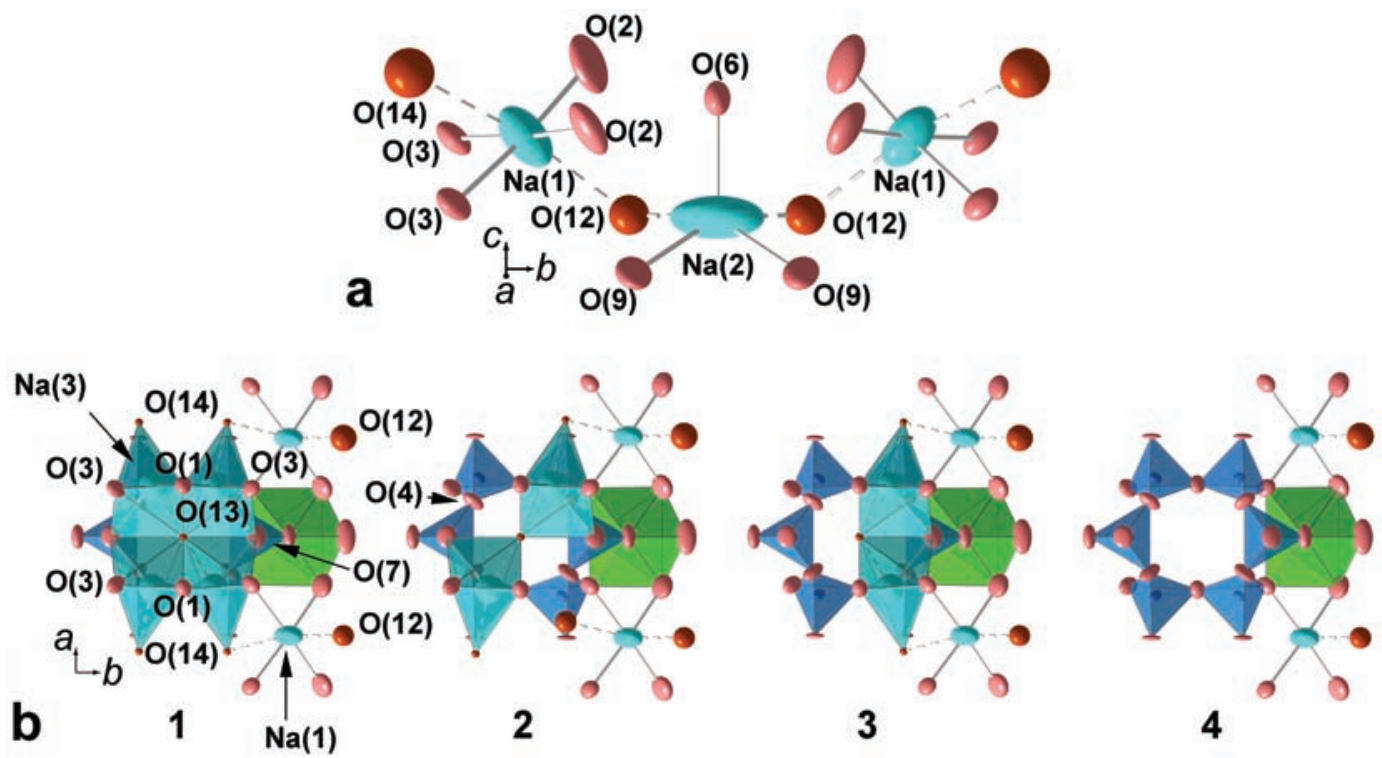

FIG. 6. The local bonding environments for the (a) $\mathrm{Na}(1)$ and $\mathrm{Na}(2)$ sites and $(b)$ the $\mathrm{Na}(3)$ sites. In (b) different local configurations for the $\mathrm{Na}(3)$ site are shown: while configuration 1 is not allowed because of very short $\mathrm{Na}(3)-\mathrm{Na}(3)$ distances $(2.31 \AA)$; all the other cases are possible.

local charge deficit at these anion sites due to vacancies at the adjacent $\mathrm{Na}(3)$ sites.

The substitution of $\mathrm{Ca}^{2+}$ for $R E E^{3+}$ requires a higher-valence species replacing a lower-valence species. However, $\mathrm{Ca}$ and $\mathrm{S}$ are negatively correlated. Another possibility is $\mathrm{Na} \rightarrow \square$, with the resulting substitution

$$
\mathrm{Ca}^{2+}+\mathrm{Na}^{+} \rightarrow R E E^{3+}+\square .
$$

Again, we cannot check this substitution by the corresponding variation in $\mathrm{Na}$ because of the inaccurate analysis of that element.

Some sazhinite-(La) contains significant $\mathrm{S}$, which is correlated with $\mathrm{Si}$ (Fig. 4c) suggesting that they substitute for each other. Inspection of

TABLE 8. Bond valence (vu) calculations. Formula assumed $\mathrm{Na}_{3} \mathrm{La}_{[}\left[\mathrm{Si}_{6} \mathrm{O}_{15}\right] \cdot 2 \mathrm{H}_{2} \mathrm{O}$.

\begin{tabular}{|c|c|c|c|c|c|c|c|c|c|}
\hline & $\mathrm{Si}(1)$ & $\mathrm{Si}(2)$ & $\mathrm{Si}(3)$ & $\mathrm{Si}(4)$ & $M$ & $\mathrm{Na}(1)$ & $\mathrm{Na}(2)$ & $\mathrm{Na}(3)$ & $\Sigma$ \\
\hline $\mathrm{O}(1)$ & $0.987{ }^{\times 2} \rightarrow$ & & & & & & & & 1.974 \\
\hline $\mathrm{O}(2)$ & & 1.151 & & & $0.565 \times 2 \downarrow$ & $0.195 \times 2 \downarrow$ & & & 1.911 \\
\hline $\mathrm{O}(3)$ & 1.145 & & & & $0.487 \times 2 \downarrow$ & $0.157 \times 2 \downarrow$ & $\begin{array}{l}x 2 \downarrow \\
-1\end{array}$ & 0.142 & 1.931 \\
\hline $\mathrm{O}(4)$ & 1.000 & & $1.000 \quad \times 2 \downarrow$ & & & & & 0.096 & 2.096 \\
\hline $\mathrm{O}(5)$ & & 1.000 & & $1.076 \times 2 \downarrow$ & & & & & 2.076 \\
\hline $\mathrm{O}(6)$ & & $0.971 \stackrel{\times 2}{ } \rightarrow$ & & & & & 0.192 & & 2.134 \\
\hline$O(7)$ & & & 1.154 & & 0.587 & & & 0.141 & 2.023 \\
\hline $\mathrm{O}(8)$ & & & 0.997 & 0.955 & 0.182 & & & & 2.134 \\
\hline $\mathrm{O}(9)$ & & & & 1.132 & 0.386 & & $0.198 \times 2 \downarrow$ & $\rightarrow$ & 1.914 \\
\hline $\mathrm{O}(10)$ & $1.005 \quad \times 2 \rightarrow$ & & & & & & & & 2.010 \\
\hline $\mathrm{O}(11)$ & & $0.997 \stackrel{\times 2}{\rightarrow} \rightarrow$ & & & & & & & 1.994 \\
\hline $\mathrm{O}(12)$ & & & & & & 0.106 & $0.068 \times 2 \downarrow$ & $\rightarrow$ & 0.242 \\
\hline $\mathrm{O}(13)$ & & & & & & & & $0.282 \times 2 \rightarrow$ & 0.564 \\
\hline $\mathrm{O}(14)$ & & & & & & 0.061 & & 0.181 & 0.242 \\
\hline$\Sigma$ & 4.137 & 4.119 & 4.151 & 4.239 & 3.261 & 0.871 & 0.724 & 0.842 & \\
\hline
\end{tabular}

Parameters for calculations taken from Brown and Altermatt (1985) for $\mathrm{La}^{3+}$ and $\mathrm{Na}^{+}$, and from Brese and O'Keeffe (1991) for $\mathrm{Si}^{4+}$. 
Table 5 shows that the $\langle\mathrm{Si}(4)-\mathrm{O}\rangle$ distance is $0.009 \AA$ less than the $<\mathrm{Si}(1,2,3)-\mathrm{O}>$ distance, lending support for $\mathrm{S}$ at the $\mathrm{Si}(4)$ site. The chemical data (Table 2) show that the crystal used in the refinement has $0.06 \mathrm{~S}$ a.p.f.u. The empirical radii of $\mathrm{Si}^{4+}$ and $\mathrm{S}^{6+}$ are 0.26 and $0.12 \AA$, respectively, and hence we expect that replacement of $0.06 \mathrm{Si}^{4+}$ by $\mathrm{S}^{6+}$ will reduce the mean bond length by $(0.26-0.12) \times 0.06=0.008 \AA$, in good accord with the observed value. Thus, $\mathrm{S}^{6+}$ probably replaces $\mathrm{Si}^{4+}$ at the $\mathrm{Si}(4)$ site. The charge imbalance introduced by this substitution can, once again, be compensated for by the replacement of $\mathrm{Na}$ according to $\mathrm{S}^{6+}+2 \square \rightarrow \mathrm{Si}^{4+}+2 \mathrm{Na}^{+}$.

Chondrite-normalized patterns of the Aris and Lovozero (Es'kova et al., 1974) sazhinites and the Aris phonolite (von Knorring and Franke, 1987), show a linear decrease in REE from La toward the HREEs (Fig. 7). While the single-crystal pattern shows depletion of $\mathrm{Zr}$, Ti and, to a lesser extent, $\mathrm{Nb}$, the whole-rock pattern is less depleted in those elements. The depletion of these elements in sazhinite-(La) from Aris is caused by competition from the concomitant growth of other minerals such as eudialyte and korobitsynite, which deplete the fluid in small-radius cations $(<0.8 \AA)$.

\section{Related structures}

Haile and Wuensch (1997) discuss the topology of $M \mathrm{Si}_{6} \mathrm{O}_{15}$ structures, many of which contain topologically different silicate layers from that of sazhinite. The majority of the $M \mathrm{Si}_{6} \mathrm{O}_{15}$ compounds are double-chain silicates [emeleusite, $\mathrm{NaNaLiFe}^{3+}\left[\mathrm{Si}_{6} \mathrm{O}_{15}\right]$, Upton et al. 1978; tuhualite, $\mathrm{Na} \mathrm{Fe}{ }^{2+} \mathrm{Fe}^{3+}\left[\mathrm{Si}_{6} \mathrm{O}_{15}\right]$, Merlino, 1969; zektzerite, $\square \mathrm{NaLiZr}\left[\mathrm{Si}_{6} \mathrm{O}_{15}\right]$, Ghose and Wan, 1978], double-ring silicates [the milarite-group, Forbes et al. 1972, Hawthorne et al. 1991; moskvinite-(Y), $\mathrm{Na}_{2} \mathrm{~K}(\mathrm{Y}, R E E)\left[\mathrm{Si}_{6} \mathrm{O}_{15}\right]$, Sokolova et al., 2003], and a framework silicate $\left(\mathrm{K}_{2} \mathrm{Ce}\left[\mathrm{Si}_{6} \mathrm{O}_{15}\right]\right.$; Karpov et al., 1976). Haile et al. (1995) proposed that the topology of the silicate units in $\left[\mathrm{Si}_{6} \mathrm{O}_{15}\right]$ compounds is controlled by the electronegativity of the cation at the $M$ site rather than by difference in radius.

Sazhinite and the synthetic compound $\beta-\mathrm{K}_{3} \mathrm{NdSi}_{6} \mathrm{O}_{15}$ (Haile and Wuensch, 2000) have $\left[\mathrm{Si}_{6} \mathrm{O}_{15}\right]^{6-}$ sheets that are topologically equivalent; however, the positions of cations in the channels present in the $R E E-\left[\mathrm{Si}_{6} \mathrm{O}_{15}\right]^{6-}$ framework are different. Small cations $(\mathrm{Na})$ coordinate $\mathrm{H}_{2} \mathrm{O}$ groups in sazhinite, whereas larger cations (K) in $\beta-\mathrm{K}_{3} \mathrm{NdSi}_{6} \mathrm{O}_{15}$ occupy the position of the $\mathrm{H}_{2} \mathrm{O}$ group in sazhinite, similarly to that proposed for $\mathrm{Na}$ and $\mathrm{Cs}$ in the analcime-pollucite solidsolution (Armbruster and Guenter, 2001). The possibility of complete substitution of $\mathrm{Cs}$ for $\mathrm{Na}$ (plus $\mathrm{H}_{2} \mathrm{O}$ ) can thus also be inferred in sazhinite, although it would probably occupy the position of $\mathrm{K}$ atoms in the synthetic compound $\beta-\mathrm{K}_{3} \mathrm{Nd}\left[\mathrm{Si}_{6} \mathrm{O}_{15}\right]$.

Significant ionic conductivity has been suggested for $\mathrm{Na}_{3} \mathrm{Y}\left[\mathrm{Si}_{6} \mathrm{O}_{15}\right]$ (Haile et al. 1995)

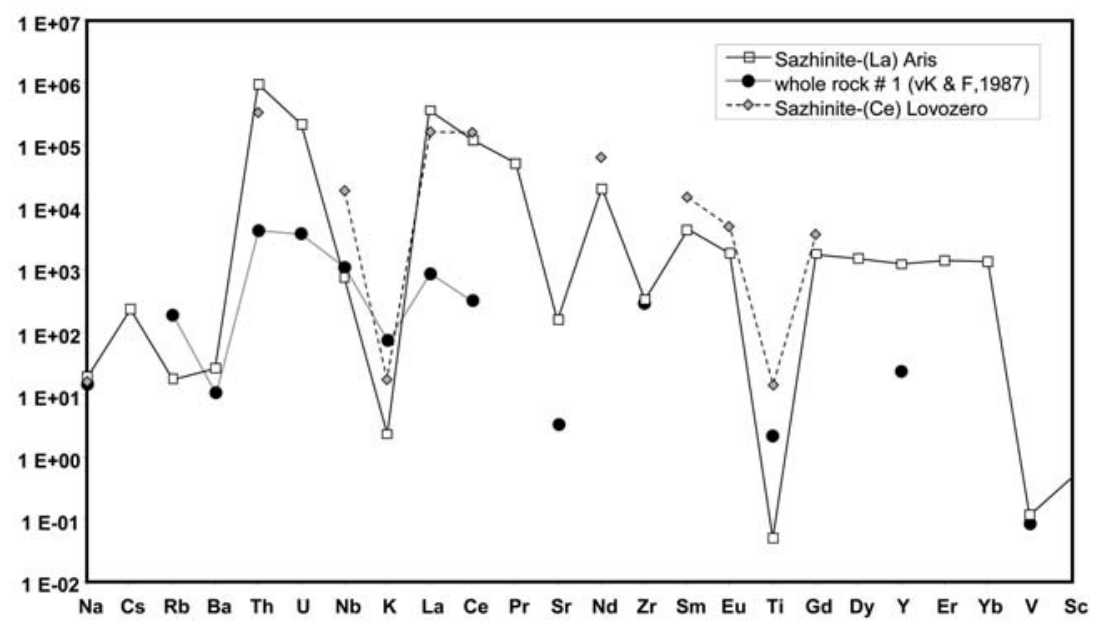

FIG. 7. Chondrite-normalized pattern of crystal 1 (point $\mathrm{A}=$ open squares). Whole-rock composition of Aris phonolite (von Knorring and Franke, 1987; filled dots) and data for sazhinite (Ce) Lovozero (Es'kova et al., 1974) (grey diamonds). 
and $\beta-\mathrm{K}_{3} \mathrm{Nd}\left[\mathrm{Si}_{6} \mathrm{O}_{15}\right]$ (Haile and Wuensch 2000). In $\mathrm{Na}_{3} \mathrm{Y}\left[\mathrm{Si}_{6} \mathrm{O}_{15}\right]$ the $\mathrm{Na}(2)$ atoms occupy half the $8 i$ site $\left(x y^{1 / 4}\right)$ where there is only $1.201 \AA$ between adjacent $\mathrm{Na}(2)$ sites. In sazhinite-(La), the $\mathrm{Na}(3)$ atoms occupy half the $4 i$ site $(x y z)$ and there is $2.312 \AA$ between two adjacent $\mathrm{Na}(3)$ sites and hopping of $\mathrm{Na}$ from occupied to unoccupied $\mathrm{Na}(3)$ sites is possible, explaining the high anisotropic displacement of the $\mathrm{H}_{2} \mathrm{O}$ groups at the $\mathrm{O}(14)$ sites. If this effect propagates then ionic conductivity is possible along the $a$ axis.

\section{Acknowledgements}

We thank R. Gastoni, M. Palenzona, M. Veschambre, and J.-L. Devidal for preparation of the EMPA-SIMS mounts and for technical assistance with the ion- and electron probe. The Consiglio Nazionale delle Ricerche is acknowledged for financing the ion microprobe at IGG (Pavia). FCH was supported by a Canada Research Chair and by a Discovery grant from the National Sciences and Engineering Research Council of Canada. D. Shannon kindly provided the sazhinite samples. LAJG acknowledges funding from the National Science Foundation (EAR-0418960). Review by M.D. Welch is acknowledged.

\section{References}

Armbruster, T. and Gunter, M.E. (2001) Crystal structures of natural zeolites. Pp. 1-67 in: Natural Zeolites; Occurrence, Properties Applications (D.L. Bish and D.W. Ming, editors). Reviews in Mineralogy and Geochemistry, 45, Mineralogical Society of America, Washington D.C.

Bartelmehs, K.L., Bloss, F.D., Downs, R.T and Birch, J.B. (1992) EXCALIBR II. Zeitschrift für Kristallographie, 199, 185-196.

Brown, I.D. and Altermatt, D. (1985) Bond-valence parameters obtained from a systematic analysis of the Inorganic Crystal Structure Database. Acta Crystallographica, B41, 244-247.

Brese, N.E. and O'Keeffe, M. (1991) Bond-valence parameters for solids. Acta Crystallographica, B47, 192-197.

Cámara, F., Garvie, L.A.J., Devouard, B., Groy, T.L. and Buseck, P.R. (2002) The structure of Mn-rich tuperssuatsiaite: a palygorskite-related mineral. American Mineralogist, 87, 1458-1463.

Chao, G.Y., Grice, J.D. and Gault, R.A. (1991) Silinaite - a new sodium lithium silicate hydrate mineral from Mont Saint-Hilaire, Québec. The Canadian Mineralogist, 29, 359-362.
Es'kova, E.M., Semenov, E.I., Khomyakov, A.P., Kazakova, M.E. and Shumyatskaya, N.G. (1974) [Sazhinite, a new silicate of sodium and rare earths]. Zapiski Vsesoyuznogo Mineralogicheskogo Obshchestva, 103, 338-341.

Fleet, S.G. (1965) The crystal structure of dalyite. Zeitschrift für Kristallographie, 121, 349-368.

Forbes, W.C., Baur, W.H. and Khan, A.A. (1972) Crystal chemistry of milarite-type minerals. American Mineralogist, 57, 463-472.

Garvie, L.A.J., Devouard, B., Groy, T.L., Cámara, F. and Buseck, P.R. (1999) Crystal structure of kanemite, $\mathrm{NaHSi}_{2} \mathrm{O}_{5} \cdot 3 \mathrm{H}_{2} \mathrm{O}$, from the Aris phonolite, Namibia. American Mineralogist, 84, 1170-1175.

Ghose, S. and Wan, Che'ng (1978) Zektzerite, $\mathrm{NaLiZrSi}_{6} \mathrm{O}_{15}$ : a silicate with six-tetrahedral-repeat double chains. American Mineralogist, 63, 304-310.

Haile, S.M. and Wuensch, B.J. (1997) Comparison of the crystal chemistry of selected $\mathrm{MSi}_{6} \mathrm{O}_{15}$-based silicates. American Mineralogist, 82, 1141-1149.

Haile, S.M. and Wuensch, B.J. (2000) Structure, phase transitions and ionic conductivity of $\mathrm{K}_{3} \mathrm{NdSi}_{6} \mathrm{O}_{15} \cdot x \mathrm{H}_{2} \mathrm{O}$. II. Structure of $\beta-\mathrm{K}_{3} \mathrm{NdSi}_{6} \mathrm{O}_{15}$. Acta Crystallographica, B56, 349-362.

Haile, S.M., Maier, J., Wuensch, B.J. and Laudise, R.A. (1995) Structure of $\mathrm{Na}_{3} \mathrm{YSi}_{6} \mathrm{O}_{15}$ - a unique silicate based on discrete $\mathrm{Si}_{6} \mathrm{O}_{15}$ units, and a possible fastion conductor. Acta Crystallographica, B51, $673-680$.

Hawthorne, F.C., Kimata, M., Černý, P., Ball, N., Rossman, G.R. and Grice, J.D. (1991) The crystal chemistry of the milarite-group minerals. American Mineralogist, 76, 1836-1856.

Horváth, L. and Gault, R.A. (1990) Toute la minéralogie sur le Mont St Hilaire (Canada). Mineralogical Record, 21(4), 284-368.

Horváth, L., Pfenninger-Horváth, E., Gault, R.A. and Tarassoff, P. (1998) Mineralogy of the Saint-Amable Sill; Varennes and Saint-Amable, Quebec. Mineralogical Record, 29(2), 83-118.

Karpov, O.G., Podeminskaya, E.A. and Belov, N.E. (1976) Crystal structure of a K, Ce silicate with a three-dimensional anion framework: $\mathrm{K}_{2} \mathrm{Ce} \mathrm{Si}_{6} \mathrm{O}_{15}$. Soviet Physics Crystallography, 22, 382-384.

Mandarino, J.A. (1981) The Gladstone-Dale relationship: Part IV. The compatibility concept and its application. The Canadian Mineralogist, 19, 441-450.

Merlino, S. (1969) Tuhualite crystal structure. Science, 166, 1399-1401.

Niedermayr, G., Gault, R.A., Petersen, O.V. and Brandstätter, F. (2002) Korobitsynite from the Aris phonolites, Windhoek, Namibia. Neues Jahrbuch für Mineralogie, 42-48.

Ottolini, L., Cámara, F. and Devouard, B. (2004) New SIMS procedures for the characterization of a 


\section{F. CÁMARA ET AL.}

complex silicate matrix, $\mathrm{Na}_{3}(\mathrm{REE}, \mathrm{Th}, \mathrm{Ca}, \mathrm{U})$ $\mathrm{Si}_{6} \mathrm{O}_{15} \cdot 2.5 \mathrm{H}_{2} \mathrm{O}$ (sazhinite), and comparison with EMPA and SREF results. Microchimica Acta, $\underline{\mathbf{1 4 5}}$, 139-146.

Sheldrick, G.M. (1996) SADABS, Siemens area detector absorption correction software. University of Göttingen, Germany.

Sokolova, E., Hawthorne, F.C., Agakhanov, A.A. and Pautov, L.A. (2003) The crystal structure of moskvinite-(Y), $\mathrm{Na}_{2} \mathrm{~K}(\mathrm{Y}, \mathrm{REE})\left[\mathrm{Si}_{6} \mathrm{O}_{15}\right]$, a new silicate mineral with $\left[\mathrm{Si}_{6} \mathrm{O}_{15}\right]$ three-membered double rings from the Dara-i-Pioz moraine, TienShan mountains, Tajikistan. The Canadian Mineralogist, 41, 513-520.

Shumyatskaya, N.G., Voronkov, A.A. and Pyatenko, Ya.A. (1980) Sazhinite, $\mathrm{Na}_{2} \mathrm{Ce}\left[\mathrm{Si}_{6} \mathrm{O}_{14}(\mathrm{OH})\right] \cdot \mathrm{nH}_{2} \mathrm{O}$ : a new representative of the dalyite family in crystal chemistry. Soviet Physics Crystallography, 25,
419-423.

Upton, B.G.J., Hill, P.G., Hohnsen, O. and Petersen, O.V. (1978) Emeleusite: a new $\mathrm{LiNaFe}^{\mathrm{III}}$ silicate from South Greenland. Mineralogical Magazine, 42, $31-34$.

von Knorring, O. and Franke, W. (1987) A preliminary note on the mineralogy and geochemistry of the Aris phonolite, SWA/Namibia. Communications of the Geological Survey of S.W. Africa/Namibia, 3, 61.

von Knorring, O., Petersen, O.V., Karup-Møller, S., Leonardsen, E.S. and Condliffe, E. (1992) Tuperssuatsiaite from Aris phonolite, Windhoek, Namibia. Neues Jahrbuch für Mineralogie Monatshefte, 145-152.

[Manuscript received 7 October 2005:

revised 3 August 2006] 